\title{
Imaging nanostructures with coherent phonon pulses
}

\author{
B. C. Daly, ${ }^{a}$ N. C. R. Holme, T. Buma, C. Branciard, and T. B. Norris \\ Center for Ultrafast Optical Science and EECS Department, University of Michigan, Ann Arbor, \\ Michigan 48109-2099
}

D. M. Tennant, J. A. Taylor, J. E. Bower, and S. Pau

Bell Laboratories, Lucent Technologies Murray Hill, New Jersey 07974

(Received 4 February 2004; accepted 26 April 2004; published online 10 June 2004)

\begin{abstract}
We demonstrate submicron resolution imaging using picosecond acoustic phonon pulses. High-frequency acoustic pulses are generated by impulsive thermoelastic excitation of a patterned 15-nm-thick metal film on a crystalline substrate using ultrafast optical pulses. The spatiotemporal diffracted acoustic strain field is measured on the opposite side of the substrate, and this field is used in a time-reversal algorithm to reconstruct the object. The image resolution is characterized using lithographically defined 1-micron-period Al structures on Si. Straightforward technical improvements should lead to resolution approaching $45 \mathrm{~nm}$, extending the resolution of acoustic microscopy into the nanoscale regime. () 2004 American Institute of Physics. [DOI: $10.1063 / 1.1764599]$
\end{abstract}

Progress in nanoscience is facilitated by advances in nanoscale imaging. In this letter, we describe a technique that enables acoustic microscopy to be performed in the submicron domain, with the potential to obtain a resolution of roughly $45 \mathrm{~nm}$. State-of-the-art acousitc microscopes have a maximum resolution in the range of $1-10$ microns, and use sound waves with frequencies as high as $1 \mathrm{GHz} .{ }^{1}$ We propose to improve this resolution dramatically by using considerably higher frequency $(>100 \mathrm{GHz})$ acoustic waves which are generated and detected by means of an ultrafast optical pump and probe technique known as picosecond ultrasonics. $^{2-4}$

In picosecond ultrasonics, an ultrafast (typically $100 \mathrm{fs}$ ) laser pulse impulsively heats a thin (typically 10-nm-thick) metal film, and the resulting thermal expansion launches a single-cycle coherent acoustic phonon pulse into the substrate. This technique has become a powerful tool for nanometer-scale thin-film metrology and semiconductor process control. ${ }^{4}$ We emphasize here, however, that picosecond ultrasonics is capable of imaging two-dimensional (2D) lateral nanostructures and potentially three-dimensional (3D) nanostructures as well. To accomplish this, high-resolution optical measurement of the transverse spatial variation of the acoustic wave is required. Our solution is to allow the scattered (diffracted) acoustic wave to propagate a sufficient distance such that this variation is broad enough to be measured by a 1 micron diameter optical probe beam. Unfortunately, the ballistic propagation length of terahertz-bandwidth acoustic phonons is typically only a few microns, roughly two orders of magnitude shorter than the diffraction length required for our method. However, it has been shown that coherent phonon pulses can propagate for several $\mathrm{mm}$ in crystalline substrates such as $\mathrm{Si}$ or sapphire at cryogenic temperatures. ${ }^{5-7}$

The concept underlying our imaging method is illustrated in Fig. 1. Input pulses are scattered from the object plane $\Sigma$ and time domain measurements of the scattered field amplitude $u\left(P_{0}, t\right)$ are made at a number of different detector

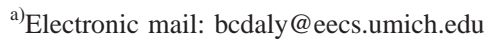

positions $P_{0}$ on a surface $\Sigma^{\prime}$ in the far field. If the field on the surface $\Sigma^{\prime}$ is then time reversed, it will "backpropagate" to the object plane due to the time-reversal symmetry of the wave equation. The sum of these numerically backpropagated waves yields a reconstruction of the input field at the object plane, and therefore of the object itself. Thus, the object can be reconstructed by using the measured field as the input to the "time-reversed" diffraction integral, ${ }^{8}$

$$
\begin{aligned}
u\left(P_{1}, t\right)= & -\frac{1}{4 \pi c} \iint_{\Sigma^{\prime}}\left(\frac{1+\cos \left(\mathbf{n}_{0}, \mathbf{r}_{01}\right)}{\mathbf{r}_{01}}\right) \frac{\partial}{\partial t} u \\
& \times\left(P_{0}, t+\frac{\mathbf{r}_{01}}{c}\right) d \sigma^{\prime},
\end{aligned}
$$

where $c$ is the wave velocity, $\mathbf{n}_{0}$ is the normal to the far-field surface, and $\mathbf{r}_{01}$ is the vector between $P_{0}$ a point $P_{1}$ in the object plane. We refer to this method as "time-reversal imaging," and have previously demonstrated its applicability to both 2D (Ref. 9) and 3D (Ref. 10) imaging with single-cycle terahertz electromagnetic pulses.

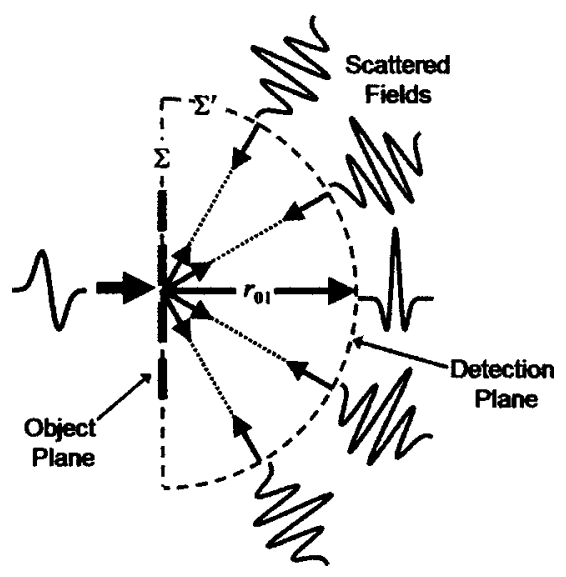

FIG. 1. Illustration of the basic time-reversal imaging concept. The scattered fields at the detection screen $\Sigma^{\prime}$ are used as the input to Eq. (1) in order to backpropagate the waves to the input aperture plane $\Sigma$. 

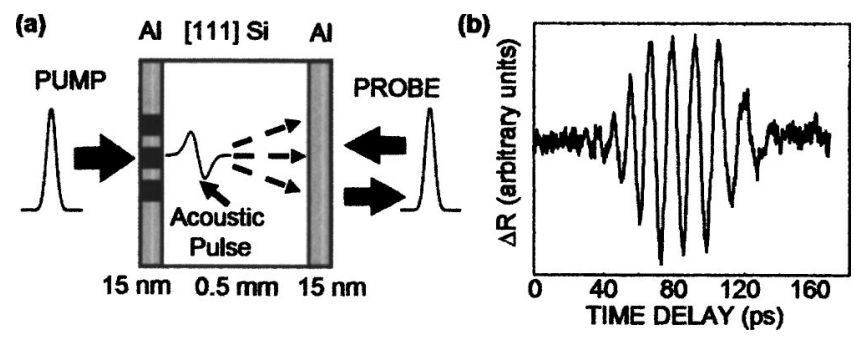

FIG. 2. (a) Illustration of the pump and probe imaging experiment (close-up of sample in cryostat). (b) Change in reflectivity vs delay time for a single probe spot at $20 \mu \mathrm{m}$ off axis.

To perform time-reversal imaging with picosecond ultrasonic pulses, it is necessary that the acoustic field diffract sufficiently so that it can be measured with a focused optical probe beam. In a recent paper, we investigated the effects of diffraction on picosecond ultrasonic pulses in a crystalline [111] Si substrate. ${ }^{11}$ We found that when the acoustic source is small (radius $\sim 3 \mu \mathrm{m}$ ), and the propagation distance is large enough $(\sim 0.5 \mathrm{~mm})$, the acoustic pulses diffract into the far field. This was manifested by a change in the acoustic pulse shape caused by a diffraction-induced axial phase advance (Gouy phase shift). ${ }^{12-14}$ This observation is an excellent indicator of our ability to resolve transverse spatial variations in the propagating acoustic field.

A simple illustration of the imaging scheme is given in Fig. 2(a). Optical pump pulses $(200-300 \mathrm{fs}, 810 \mathrm{~nm}, 50 \mathrm{pJ})$ derived from a mode-locked, $76 \mathrm{MHz}$ repetition rate, Ti:Sapphire laser are used to heat $\left(\Delta T_{\mathrm{FILM}} \sim 10 \mathrm{~K}\right.$ for an individual pulse) a patterned 15-nm-Al film on a high-purity [111] $\mathrm{Si}$ substrate. The sample is kept at a temperature below $20 \mathrm{~K}$ in order to minimize attenuation due to phonon-phonon scattering in the Si. For a substrate with a high thermal conductivity, such as $\mathrm{Si}$, the steady-state heating of the film is negligible. ${ }^{15}$ We used electron-beam lithography to etch the $\mathrm{Al}$ film with lines that are $1 \mu \mathrm{m}$ in width. Note that since the Al lines are illustrated by the optical pump beam (radius $\sim 3 \mu \mathrm{m}$ ), the object to be imaged is now the source of our detected waves. The optically induced thermal expansion of the $\mathrm{Al}$ film produces roughly single cycle strain pulses composed of longitudinal acoustic phonons with a peak frequency and bandwidth of $\sim 100 \mathrm{GHz}$, and an amplitude of the order of $1 \times 10^{-4}$. $^{2}$ The relatively small acoustic mismatch $(\sim 20 \%)$ between $\mathrm{Al}$ and $\mathrm{Si}$ allows most of this strain to propagate into the Si. $100 \mathrm{GHz}$ corresponds to an acoustic wavelength in [111] Si of $\sim 90 \mathrm{~nm}$; thus, we ultimately expect this system to achieve a diffraction limited resolution of $\sim 45 \mathrm{~nm}$. The acoustic pulses propagate to a second $\mathrm{Al}$ transducer on the other side of the Si substrate. There, a slight change in reflectivity of the order of $1 \times 10^{-5}$ induced by the time varying positions by an optical probe beam (radius $\sim 0.5 \mu \mathrm{m}$ ). The amplitude of the strain field at each position can, therefore, be measured in the time domain simply by varying the time delay between the optical pump and probe pulses. Figure 2(b) shows an example of the raw reflectivity data versus time delay for one particular probe spot located 20 microns off axis in a direction perpendicular to the lines. The multiple peaks are indicative of the acoustic waves arriving from the different lines illuminated by the pump spot.

At this point, we could simply input the detected acoustic wave field into a numerical backpropagation algorithm based on Eq. (1) in order to calculate a $2 \mathrm{D}$ profile of the

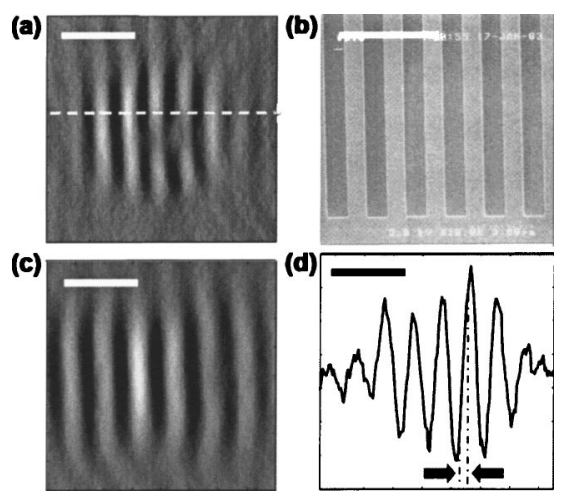

FIG. 3. $1 \mu \mathrm{m}$ lines. The scale bar in the top left corner of each picture represents a length of $5 \mu \mathrm{m}$. (a) Reconstructed acoustic image of the $\mathrm{Al}$ lines (from data). The image shown is a linear gray-scale plot. The dashed white line indicates the line out used for (d). (b) SEM image of the Al lines. Note that the scale is different for this picture than for the others. (c) Reconstructed image of the Al lines from the same data set that produced (a) but without the anisotropic group velocity. (d) Line out of the reconstruction amplitude vs position for the data shown in (a). The distance (indicated by the arrows) between the $10 \%$ (peak-to-peak value) point and the $90 \%$ point for the edge of the line indicated in the plot is $\sim 700 \mathrm{~nm}$.

initial acoustic field at the patterned Al transducer. Unfortunately, this would be incorrect due to the elastic anisotropy of the crystalline Si wafer. Since the velocity of sound wave propagation in crystalline solids is dependent on the propagation direction, it is necessary to rewrite the velocity $c$ in Eq. (1) with an angular dependence [i.e., $c \equiv c(\theta, \varphi)]$. Furthermore, when discussing the propagation of acoustic phonons in anisotropic media, it is important to distinguish between the phase velocity and the group velocity. From anisotropic elasticity theory, we know that even in a medium with no dispersion, the group velocity can still deviate from the phase velocity in both magnitude and direction. ${ }^{16,17}$ This is because the 3D group velocity is given by the gradient of a surface of constant frequency in wave vector space:

$$
\mathbf{c}_{G}=d \omega(\mathbf{k}) / d \mathbf{k}=\nabla_{k} \omega(\mathbf{k}) .
$$

For light propagating in a vacuum or for sound propagating in an elastically isotropic material, $\omega(\mathbf{k})$ is simply spherical, and the group velocity has the same direction as the phase velocity. However, in an anisotropic crystal such as $\mathrm{Si}, \omega(\mathbf{k})$ deviates significantly from a sphere, resulting in a group velocity whose direction disagrees with the phase velocity. This effect is known as "phonon focusing." 18 To correctly reconstruct our object, we must use this group velocity $c_{G}$ in place of $c$ in Eq. (1).

Figure 3(a) shows the reconstructed acoustic image of the $\mathrm{Al}$ pattern along with a scanning electron microscope (SEM) image of the sample (Fig. 3(b)). The wave field was measured at 80 equidistant points along the perimeter of a $40 \mu \mathrm{m} \times 40 \mu \mathrm{m}$ square. While the image is not perfect, the reproduction of the region of the $\mathrm{Al}$ pattern illuminated by the pump spot is unmistakable. Figure 3(c) shows the reconstructed image when the effects of anisotropy described above are ignored. Clearly, the image is greatly distorted and the number of reconstructed lines is inconsistent with the known pump spot size $(r \sim 3 \mu \mathrm{m})$. Figure 3(d) shows a lineout from the reconstruction in Fig. 3(a) which gives an estimate of the resolution of the current experimental configuration. For the edge of the reconstructed line indicated on the plot, we find that the distance from $10 \%$ to $90 \%$ of the maxi- 
(a)
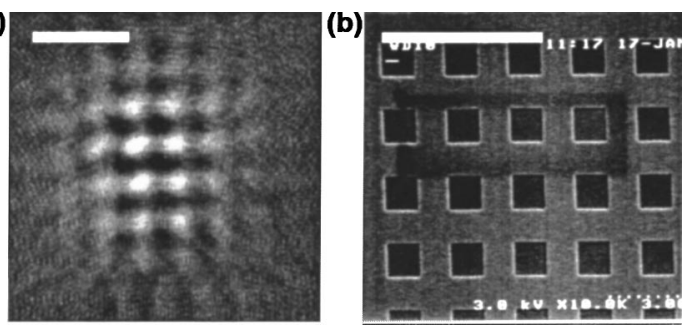

FIG. 4. $1 \mu m$ grid. The scale bar in the top left corner of each picture represents a length of $5 \mu \mathrm{m}$. (a) Reconstructed acoustic image. (b) SEM image. Note that the scale is different for the two images.

mum reconstructed peak-to-peak amplitude yields a resolution width of approximately $700 \mathrm{~nm}$, indicating that submicron resolution is achievable with this scheme. In Fig. 4(a), we show an image obtained from a 2D grid structure (again with 1 micron lines), along with an SEM image of the same structure [Fig. 4(b)].

Several factors limit the resolution of the technique in our experiment. First, the main technical limitation is vibration of the sample mount in the helium-flow cryostat. Computer simulations of the imaging experiment for this particular sample indicate that suppression of these vibrations will enable us to achieve about $200 \mathrm{~nm}$ resolution. The second limitation is geometrical-specifically the finite spot size of our optical probe beam. The spatial resolution of our image is critically dependent on our ability to temporally resolve the acoustic pulses arriving from different parts of the $\mathrm{Al}$ pattern. Therefore, an excessively large optical probe spot size will cause a temporal averaging of those signals (i.e., a washing out of the peaks). Currently our optical probe spot has a radius of $\sim 0.5$ microns, close to the diffraction limit for $810 \mathrm{~nm}$ light. Switching to a shorter-wavelength probe would be one way to improve this figure. Additionally, our flat detection plane leads to an effective increase of the detection aperture as one moves the probe spot further and further off axis. Unfortunately, in order to achieve maximum resolution, the off-axis probe displacement must be considerably larger than the case described in this letter. A spherical detection surface (as illustrated in Fig. 1) would greatly reduce this obliquity effect, enabling $45 \mathrm{~nm}$ resolution to be obtained (such surfaces can be obtained on Si using greyscale lithography techniques). ${ }^{19}$
Nanoscale acoustic imaging is expected to provide a tool for the characterization of nanomaterials and nanoelectronic devices, and potentially for acoustic cryomicroscopy of biological samples. One advantage of this approach is that it provides an additional image contrast mechanism (elastic properties) for the expanding field of nanoscale imaging. Additionally, although our proof-of-principle experiments imaged an object on the substrate surface, our approach should be able to image structures below the surface as well. Such embedded objects are not accessible by conventional metrology techniques without destroying the sample. Finally, this technique can, in principle, provide 3D images, and thus may serve as a complementary technique to more standard surface-imaging nanoprobes.

This work was supported by AFOSR through the MURI program, and by the Frontiers in Optical Coherent and Ultrafast Science (FOCUS) NSF Physics Frontiers Center.

${ }^{1}$ A. Briggs, Advances in Acoustic Microscopy (Plenum, New York, 1995).

${ }^{2}$ C. Thomsen, H. T. Grahn, H. J. Maris, and J. Tauc, Phys. Rev. B 34, 4129 (1986).

${ }^{3}$ H. Grahn, H. Maris, and J. Tauc, IEEE J. Quantum Electron. 25, 2562 (1989).

${ }^{4}$ H. Maris, Sci. Am. 278, 86 (1998).

${ }^{5}$ H. Y. Hao and H. J. Maris, Phys. Rev. Lett. 84, 5556 (2000).

${ }^{6}$ H. Y. Hao and H. J. Maris, Phys. Rev. B 64, 064302 (2001).

${ }^{7}$ O. L. Muskens and J. I. Dijkhuis, Phys. Rev. Lett. 89, 285504 (2002).

${ }^{8}$ A. B. Ruffin, J. Decker, L. Sanchez-Palencia, L. LeHors, J. F. Whitaker, T. B. Norris, and J. V. Rudd, Opt. Lett. 26, 681 (2001).

${ }^{9}$ A. B. Ruffin, J. Van Rudd, J. Decker, L. Sanchez-Palencia, L. LeHors, J. F. Whitaker, and T. B. Norris, IEEE J. Quantum Electron. 38, 1110 (2002).

${ }^{10}$ T. Buma and T. B. Norris, Appl. Phys. Lett. 84, 2196 (2004).

${ }^{11}$ N. C. R. Holme, B. C. Daly, M. T. Myaing, and T. B. Norris, Appl. Phys. Lett. 83, 292 (2003).

${ }^{12}$ A. E. Siegman, Lasers (University Science Books, Sausalito, CA, 1986), Chap. 17

${ }^{13}$ S. Feng and H. G. Winful, Opt. Lett. 26, 485 (2001).

${ }^{14}$ A. B. Ruffin, J. V. Rudd, J. F. Whitaker, S. Feng, and H. G. Winful, Phys. Rev. Lett. 84, 3410 (1999).

${ }^{15}$ W. S. Capinski and H. J. Maris, Rev. Sci. Instrum. 67, 2720 (1996).

${ }^{16}$ J. P. Wolfe, Imaging Phonons (Cambridge University Press, New York, 1998).

${ }^{17}$ B. A. Auld, Acoustic Fields and Waves in Solids, 2nd ed. (Krieger, Malabar, FL, 1990).

${ }^{18}$ B. Taylor, H. J. Maris, and C. Elbaum, Phys. Rev. Lett. 23, 416 (1969).

${ }^{19}$ C. M. Waits, A. Modafe, and R. Ghodssi, J. Micromech. Microeng. 13, 170 (2003). 